\title{
A Case of multifocal giant rhabdomyoma in head and neck region
}

\begin{abstract}
Rhabdomyoma is a rare benign mesenchymal tumor originating from striated muscle. It is usually located in heart among children. In adulthood, it is reported most commonly between the ages of 25-40years, especially in males, and located in extracardiac regions. It is frequently located in head and neck region, mainly on tongue, base of mouth, soft palate, pharynx and larynx in adults. Since rhabdomyomas are slow growing, painless masses, the clinical picture may be asymptomatic or may change depending on the affected region. Sixty eight years old male patient was admitted to our clinic with the complaints of progressive swelling on neck and swallowing difficulty accompanying unable to close mouth properly and speech defects of 10years. Since the mass completely fulfilling mouth base, submandibular spaces and submental areas bilaterally was determined after the investigations, total mass excision was performed under general anesthesia. The clinical picture and treatment of the patient whose pathological diagnosis was rhabdomyoma is reported here with the review of the literature.
\end{abstract}

Keywords: rhabdomyoma, head and neck neoplasms, surgery
Volume 2 Issue 2 - 2015

\author{
Omer Bayir,' Tugba Karagoz,' Kemal \\ Keseroglu,' Volkan Yucel,' Bilgihan Acikgoz, ${ }^{2}$ \\ Guleser Saylam,' Banu Tijen Ceylan,' Ali \\ Ozdek,' Mehmet Hakan Korkmaz ${ }^{3}$ \\ 'Ministry of Health, Department of Otolaryngology and Head \& \\ Neck Surgery, Turkey \\ 2Ministry of Health, Department of Pathology, Turkey \\ ${ }^{3}$ Yildirim Beyazit University, Department of Otolaryngology and \\ Head \& Neck Surgery,Turkey
}

Correspondence: Omer Bayir, Ministry of Health, Department of Otolaryngology and Head \& Neck Surgery Mehmet Akif Ersoy Mh. 284 Sk. No: 7/9 Yenimahalle, Ankara,Turkey, Tel +90 50667262 87, Email bayiromer@hotmail.com

Received: January 25, 2015 | Published: February 25, 2015

\section{Introduction}

Rhabdomyoma is a rare benign mesenchymal tumor originating from striated muscle which is seen usually in cardiac region in children. However in adulthood, it is in males (M/F: 3/1), and located in extracardiac regions, especially in head and neck region with a $90 \%$ of incidence. ${ }^{1}$ If adult type rhabdomyomas are located in head and neck region, they are originated from striated muscle of $3^{\text {rd }}$ or $4^{\text {th }}$ branchial arche, spherical or lobulated in shape and $3 \mathrm{~cm}$ in dimensions in average (between $0,5-10 \mathrm{~cm}$ ). They are slowly growing, painless, commonly solitary but multifocal in $25 \%$ of cases and multi-centric in less than $5 \%$ of cases. Surgery is indicated usually if the patient has functional problem. ${ }^{2,3}$ The operation technique performed and clinical features of an advanced aged patient admitted with a mass originating from multiple foci which reached to a giant size $(15 \mathrm{~cm})$ in years is reported here with the review of the literature.

\section{Case report}

Sixty eight years old male patient was admitted to our clinic with the complaints of progressive swelling on neck which causes speech difficulty. He couldn't close his mouth properly (Figures $1 \& 2$ ). It was learnt that he was only drinking fluids but could not eat solid meals for long years. In his physical examination, a mass lesion fulfilling mouth base and all oral cavity pushing the tongue to the posterior was observed. This mass was filling all submental and submandibular regions bilaterally on neck and it were running over the secondary regions. The mass was soft in palpation and was approximately $20 \mathrm{x} 10 \mathrm{~cm}$ in dimensions. The patient's tongue movements could not be detected due to size of the mass but his flexible endoscopic examination and other ear- nose- throat examinations were normal. In magnetic resonance imaging (MRI), a conglomerated, highly contrast enhanced mass lesion with an approximately $20 \mathrm{~cm}$ diameter that was narrowing the upper airways, considerably widespread on submental region, at the $2^{\text {nd }}$ region level on bilateral parotid and submandibular regions was detected (Figure 3 ).

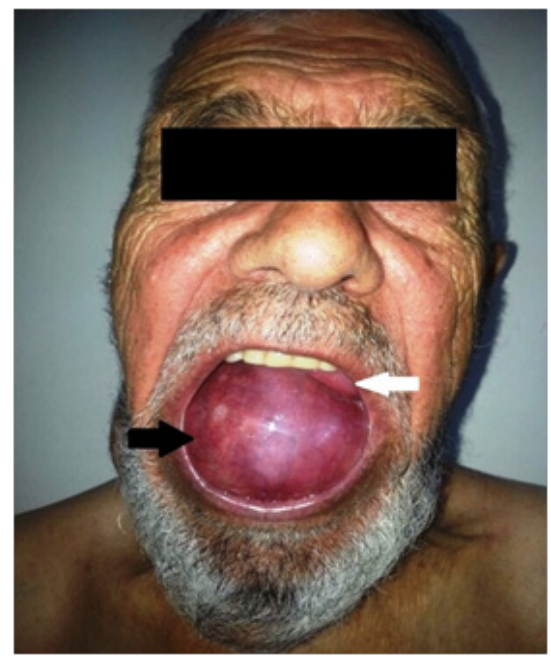

Figure I Preoperative appearance of the patient. White Arrow: Tongue; Black Arrow: Mass (Mass is fulfilling the oral cavity completely).

Intraoral incisional biopsy was performed and the diagnosis was reported as 'rhamdomyoma'. Finally, the mass of the patient was totally excised under general anesthesia (Figure 4). Peroperatively, it was observed that this pink, multilobular mass without a prominent capsule fulfilling all submandibular, submental regions and mouth base, which pushed the tongue completely to the posterior and resulted in atrophy of all tongue muscles; was originating from multiple foci on intrinsic and extrinsic tongue muscles. With the aim of exposure peroperatively, left submandibular gland excision and dissection of anterior belly of left digastric muscle were performed. Nervi hypoglossus and lingualis were preserved bilaterally. There was no postoperative complications recorded and the pathological diagnosis of the patient was rhabdomyoma. The surgical resected specimens were pieced, with a lobulated brown cut surface. Microscopically it 
was composed of tightly packed polygonal cells with a PAS positive granular or vacuolated cytoplasm. Nuclei showed vesicular chromatin, mitoses were not found. Immunohistochemically, the cells strongly expressed desmin but negative for S-100, vimentin and CD34 (Figure 5). Since minimal restriction was recorded on the tongue movements of the patient in postoperative period, swallowing rehabilitation was achieved. In postoperative period, he was able to eat solid meals together with the liquid ones and his speech was more understandable. In $1^{\text {st }}$ year follow-up after surgery no sign of relapse was present on the patient (Figure 6).

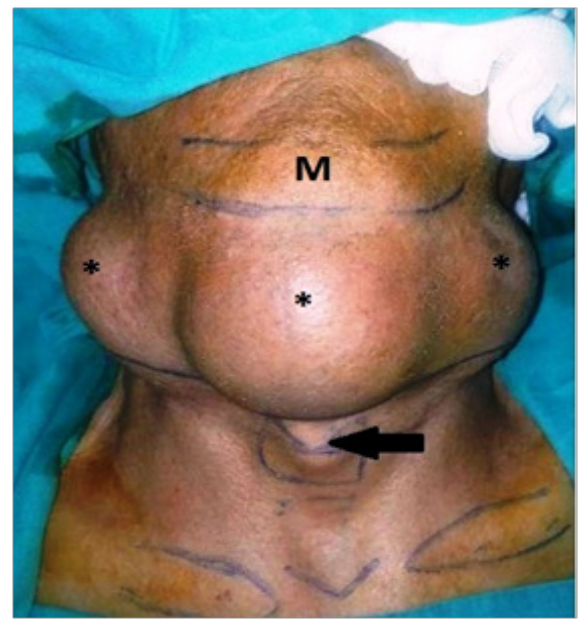

Figure 2 Preoperative appearance of the patient. black arrow, larynx, m, mentum, mass.

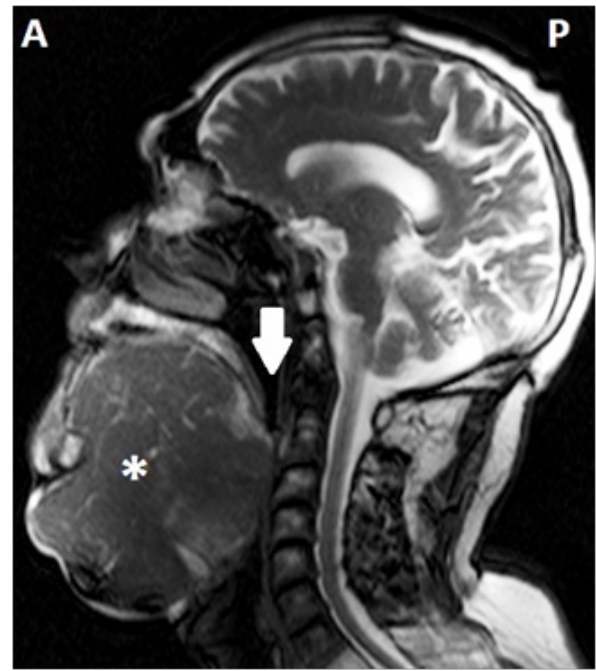

Figure 3 Sagittal TI scan MRI of the patient. A, Anterior; P, Posterior; *, Mass; White Arrow, Pharynx.

\section{Discussion}

The term rhabdomyoma was first used by Zenker in 1864 in order to define a tumor in benign character that was composed of striated muscle cells with different degrees of differentiation and maturation. ${ }^{4}$ Rhabdomyomas are classified as cardiac or extracardiac according to their localizations. Cardiac rhabdomyomas are generally reported in children and young. Adult type rhabdomyoma, which is reported at the age of 55years in average, is located in head and neck region especially on tongue, base of mouth, soft palate, pharynx and larynx.,
Some authors emphasized that they are originated from $3^{\text {rd }}$ and $4^{\text {th }}$ branchial arch muscles pathogenically. ${ }^{2}$ They very rarely originate from multiple foci. ${ }^{3}$ Although reciprocal chromosome t $(15 ; 17)$ was determined to take some place in etiology, chronic irritation is also thought to be important in pathogenesis. ${ }^{5,7}$

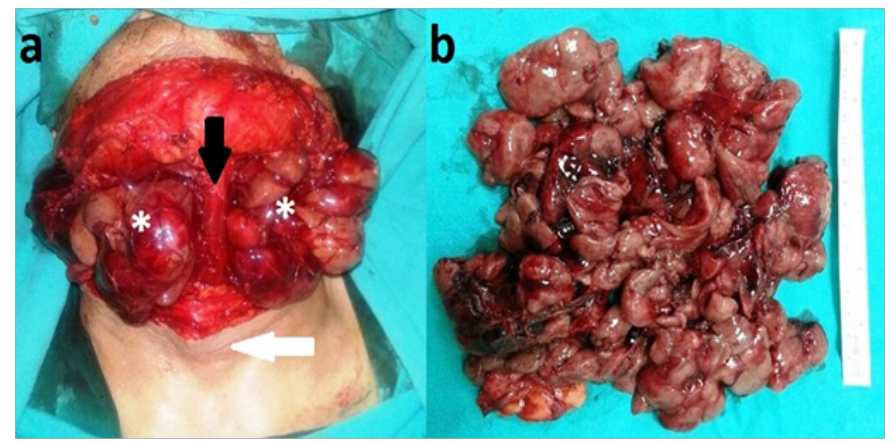

Figure 4 Preoperative appearance of the patient. A, The appearance of the mass after excision of flap. b, The appearance of mass after total excision White Arrow, Larynx; Black Arrow, Digastric Muscle; *, Mass.

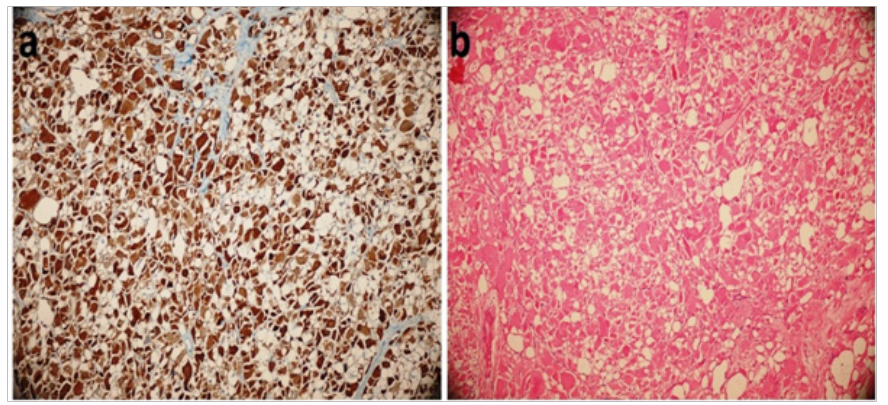

Figure 5 Light microscopy images of patient. a) Immunohistochemistry for desmin. rhabdomyoblasts show diffuse cytoplasmic reactivity (original magnification $10 \times 10)$. b) Microscopical view of the specimen, eosinophillic polygonal cells with a PAS-positive granular or vacuolated cytoplasm (hamatoxylin and eosin; original magnification I0XI0).

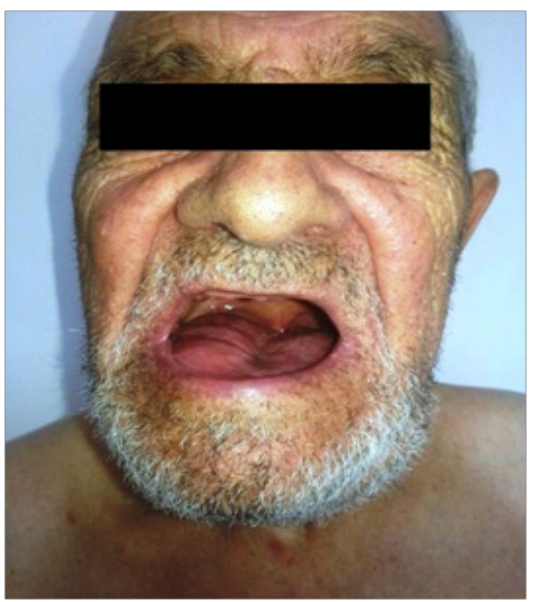

Figure 6 Postoperative Ist year appearance of the patient.

Since rhabdomyomas are painless, slow growing masses in general, they are clinically asymptomatic. With an increase in their dimensions, rhabdomyomas can cause symptoms depending on their localization. Rhabdomyomas located adjacent to the airways may result in symptoms before growing to the large masses. In differential 
diagnosis, granular cell tumor, rhabdomyosarcoma and other head and neck sarcomas and salivary gland tumors should be supposed. ${ }^{8}$

In radiologic evaluation, the appearance of rhabdomyoma is not defined clearly. In radiologic investigations, computed tomography or MRI may be preferred. Its non-invasive pattern to the contiguous tissues may help to differentiate from malign pathologies. Though it is observed as iso-intense or hyper-intense compared with normal muscle in T1 and T2 scans of MRI, this condition does not provide the obvious differentiation of rhabdomyoma from other pathologies. ${ }^{8}$

The golden standard is the surgery in treatment. Rhabdomyoma may also be apparent years after the surgical excision. The recurrence rates change between $10-42 \%$ after surgery. ${ }^{4,8}$ Moreover, rhabdomyoma may also emerge from different foci years later. ${ }^{9}$ However there is no exact data about the malign transformation in literature.

It is extremely rare in literature such a case of rhabdomyoma originating from intrinsic and extrinsic muscles of tongue and reaching huge dimensions of approximately $15 \mathrm{~cm}$ as reported here. In investigation of PubMed, approximately 20 case reports of headneck rhabdomyomas with multifocal origin were determined. In our patient, in treatment of this huge rhabdomyoma mass highly effecting life quality and esthetic appearance, total surgical excision was performed. Additionally, since the mass was huge and adjacent to important anatomical structures, the surgery was difficult to perform and vulnerable to complications. There was atrophy in tongue muscles in our patient due to the restriction of tongue movements by the mass effect for long years; this condition was markedly improved with swallowing rehabilitation. Although there was no sign of relapses in $1^{\text {st }}$ year follow up, since rhabdomyoma is a slowly growing tumor, the necessity of long time follow-up in our patient is clear.

\section{Conclusion}

In conclusion, head and neck rhabdomyomas are diseases effecting life quality and producing cosmetic deformities which are treated with total excision. Especially multifocal and huge rhabdomyomas are vulnerable to complications due to their close vicinity with important anatomical structures. Moreover, some rehabilitations such as swallowing exercises should be added to the treatment after surgery in order to increase the life quality of the patient. Since rhabdomyomas grow slowly with moderately high recurrence rates, the patients should be followed for a long time in postoperative period.

\section{Acknowledgments}

None.

\section{Conflicts of interest}

The authors declare that there are no conflicts of interest.

\section{Funding}

None.

\section{References}

1. Bjorndal Sorensen K, Godballe C, Ostergaard B, et al. Adult extracardiac rhabdomyoma: light and immunohistochemical studies of two cases in the parapharyngeal space. Head Neck. 2006;28(3):275-279.

2. Weiss S, Goldblum J. Enzinger and Weiss's soft tissue pathology. Soft Tissue Tumors. 5th edn, Mosby Inc, USA: Philadelphia; 2008. p. 584-588.

3. Hansen T, Katenkamp D. Rhabdomyoma of the head and neck: morphology and differential diagnosis. Virchows Arch. 2005;447(5):849-854.

4. de Trey LA, Schmid S, Huber GF. Multifocal adult rhabdomyoma of the head and neck manifestation in 7 locations and review of the literature. Case Rep Otolaryngol. 2013;2013:758416.

5. Gibas Z, Miettinen M. Recurrent parapharyngeal rhabdomyoma. Evidence of neoplastic nature of the tumor from cytogenetic study. Am J Surg Pathol. 1992;16(7):721-728.

6. Zachariades N, Skoura C, Sourmelis A, et al. Recurrent twin adult rhabdomyoma of the cheek. $J$ Oral Maxillofac Surg. 1994;52(12):1324-1328.

7. Pichi B, Manciocco V, Marchesi P, et al. Rhabdomyoma of the parapharyngeal space presenting with dysphagia. Dysphagia. 2008;23(2):202-204.

8. Koutsimpelas D, Weber A, Lippert BM, et al. Multifocal adult rhabdomyoma of the head and neck: a case report and literature review. Auris Nasus Larynx. 2008;35(2):313-317.

9. Vermeersch $\mathrm{H}$, van Vugt $\mathrm{P}$, Lemmerling $\mathrm{M}$, et al. Bilateral recurrent adult rhabdomyomas of the pharyngeal wall. Eur Arch Otorhinolaryngol. 2000;257(1):24-26. 